\title{
Artificial Intelligence in Personalized Medicine Application of AI Algorithms in Solving Personalized Medicine Problems
}

\author{
Jamilu Awwalu, Ali Garba Garba, Anahita Ghazvini, and Rose Atuah
}

\begin{abstract}
Artificial Intelligence has significantly gained grounds in our daily livelihood in this age of information and technology. As with any field of study, evolution takes place in terms of breakthrough or developmental research leading to advancement and friendly usability of that specific technology. Problems from different areas have been successfully solved using Artificial Intelligence algorithms. In order to use AI algorithms in solving Personalized Medicine problems such as; disease detection or prediction, accurate disease diagnosis, and treatment optimization, the choice of the algorithm influenced by its ability and applicability matters. This paper reviews the application and ability of artificial neural network (ANN), support vector machines (SVM), Naïve Bayes, and fuzzy logic in solving personalized medicine problems, and shows that the obtained results meet expectations. Also, the achievement from the previous studies encourages developers and researchers to use these algorithms in solving Medical and Personalized Medicine problems.
\end{abstract}

Index Terms-Personalized medicine, artificial neural networks, support vector machines, Naive Bayesian.

\section{INTRODUCTION}

It is always a surprising problem seeing a drug work for some people and be less effective on others, or causing side effects in another. Another problem is the question of why some people develop some diseases e.g. cancers, while others do not. Genetic make-up and other differential factors such as age; lifestyle could be reasons for these problems. As such, [1] believes medicine should approach each patient's illness as unique, with medication tailored to the person's history and biology. This approach to medical practice is known as Personalized or Precision Medicine.

Patients with same diagnostics result must not be treated the same way; they can receive different treatment in order to achieve efficient treatment as illustrated in Fig. 1. Personalized medicine as a branch or extension of Medical Sciences uses practice and medical decisions to deliver customized healthcare service to patients. The major role of personalized medicine as posited by [2] is to predict the possibility of an individual developing a disease, achieve accurate diagnosis, and optimize the best treatment available.

Manuscript received May 12, 2014; revised September 11, 2014

Jamilu Awwalu is with the Faculty of Computer Science and IT, Baze University Abuja, Nigeria (email: Jamilu.awwalu@bazeuniversity.edu.ng).

Ali Garba Garba, Anahita Ghazvini, and Rose Atuah are with the Faculty of Information Science and Technology, Universiti Kebangsaan Malaysia, 43600 Bangi, Selangor Darul Ihsan, Malaysia (e-mail: \{aligarba, P72674, roseatuah\}@siswa.ukm.edu.my).
This is accomplished with the help of genetic information, which is used as part of the baseline data in tailoring or customizing medical treatment or administration. But the majority of replicable findings do not pinpoint common genes underlying susceptibility or protection from disease; instead, current understanding centers primarily on rare genetic variants, although a number of common variants have furthered understanding as well.

The field of medicine has significantly grown over the years and emphasis is put on considering the possibility of preventing diseases by the use of modern technologies to find out the possibility of person having a disease and giving the person treatments (maybe drugs) to control the occurrence of the foreseen disease. Also with the use of technology, clinical personnel (i.e. doctors \& pharmacist) can deliver a very much efficient healthcare service as opposed to traditional techniques [3].

The use of Artificial Intelligence techniques in setting up or building personalized medicine is important in terms of precision and accuracy of disease discovery, treatment, and drug administration. The control of adverse drug reactions and enzymes metabolism which results in some people having issues eliminating drugs from their bodies, hence leading to overdose; while others eliminate the drug from the body before it gets the chance to work [4]. The use of computers in hospitals and clinics to record medical activities or use of electronic health record (EHR) systems nowadays provides medical knowledge and data that can be used as a benchmark to enhance medical service delivery.

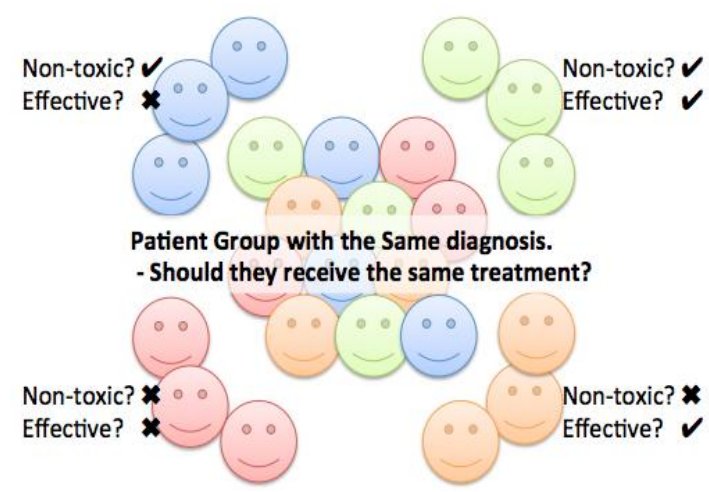

Fig. 1. Diagnosis and treatment of patients group [5].

\section{AI Algorithms Used in Personalized Medicine}

There are number of algorithms from Machine Learning and Artificial Intelligence that are used in the Medical Field, and in specific Personalized Medicine. Some of these 
algorithms are discussed here.

\section{A. Nä̈ve Bayesian}

Naïve Bayesian (NB) algorithm named after Thomas Bayes (1702-1761) who proposed the algorithm is based on probabilistic model and allows the capture of uncertainty in a model in a principled way through determining the probabilities of different outcomes. NB is widely used today in different systems that ranges from spam filtering, recommender systems, and text classification. It is also used in medical applications, and weather forecast. This makes it a good algorithm for classification and prediction as well.

Some of the advantages of NB are; it is robust for noise in input data, requires a small amount of training data. Some of the disadvantages of NB as mentioned by [6] are; loss of accuracy as a result of class conditional independence cause by assumption. And some disadvantages of Naïve Bayes, includes; where a predictor is not available from the training data, NB assumes that the record with the new predictor category has zero probability.

\section{B. Artificial Neural Network}

The application of ANNs in medicine includes, but not limited to the diagnosis, imaging, back pain, dementia, pathology and prognosis evaluation of appendicitis, myocardial infarction, acute pulmonary embolism arrhythmias, or psychiatric disorders diseases, [7] as stated by [8].

Some of the advantages of ANN as stated by [8] are: Neural networks can learn linear and non linear models. Also the accuracy of models created by neural network can be measured statistically. Incomplete data and noise are tolerable by neural network. Neural networks models are flexible because they can be updated, hence making it suitable for dynamic environment such as health sector.

ANNs are black box algorithms, hence weak in providing insight into its structure. Also, although it is able to generalize from a set of examples, if it sees only cases of a certain range; it predictions outside these range could be completely invalid [6].

\section{Support Vector Machines (SVM)}

SVM has advantages, and as mentioned by[9] they are: SVM produces accurate result classification result on theoretical basis, even when input data are non-linearly separable. Also, the accuracy result does not rely on the quality of human expertise judgment for choice of the linearization function for non linear input data.

A disadvantage of SVM as a non-parametric technique mentioned by [9] is its lack for transparency for results. The biggest limitation as mentioned by [10] is that which lies in the choice of the kernel. It has to be set correctly to achieve an accurate result for any given task or problem. Kernel choice that produces accurate results for task ' $A$ ' may produce poor results for task ' $\mathrm{B}$ '.

\section{Problems In Personalized Medicine}

The problems in personalized medicine vary and can be dependent on a disease type or nature. Overall problems from a bigger picture can be looked at from different perspectives as reported by [11] are; government laws and regulations on public medical data and genetic research, healthcare workers attitudes, awareness and education, IT implementation, and financial issues. However, the focus of this paper is on IT implementation problems, and the problems considered as shown in Fig. 2 are disease detection or prediction, achievement of accurate diagnosis, and optimal treatment.

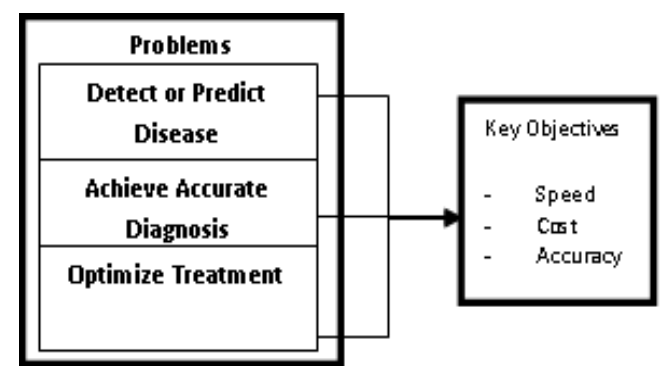

Fig. 2. Problems in personalized medicine.

\section{Application of AI TeChniques to Solve the PROBLEMS}

The problems mentioned in Fig. 2 are sequentially discussed in the subsequent sections with regards to how AI algorithms are employed by researchers to solve the problems.

\section{A. Detection/Prediction}

The solution to detection or prediction of disease is important for the solving of the next two (i.e. diagnosis and treatment) problems. In this section Artificial Intelligence algorithms from machine learning are reviewed with respect to how the problem of disease detection or prediction is solved.

\section{1) Support vector machines (SVM)}

In a study by [8], SVM was used to classify and detect fall types. The study was relevant because, falling as accident especially in the elderly aged members of the society has to be prevented; but before it can be prevented; it has to be detected and classified. Five different classifiers were used in the research, but SVM was one of two that achieved $99 \%$ accuracy.

Also, [12] used SVM to detect the accuracy of cardiac monitoring devices. The generic systems for monitoring cardiac arrests lacks the ability to differentiate between artifacts and true events across a range of individuals because they are trained just on a general population level. So, they used SVM to refine and achieve a more accurate patient specific result by making use of population level prior knowledge for initial model creation, then refining further by selectively interacting with human experts to get examples from a new patient until a stopping condition is met. The result from their study shows that patient specificity improves the Atrial Fabrillation (AF) of detection and the poor specificity of the generic detector.

\section{2) Fuzzy logic}

Case Based Fuzzy Cognitive Map (CBFCM) is an extension of fuzzy cognitive maps that is used for classification and prediction. CBFCM works by representing 
the causal relationship between different concepts and then analyzing inference patterns. This is applied in personalized medicine by analyzing the relationship between different nature of diseases and patient specific information e.g. gene, blood pressure, and blood type to infer a pattern match in the approach to disease detection in a patient.

A research by [3] on Genomic and Personalized Medicine for classification as a means of disease detection in the Decision Support System they developed. For each patient, they used three parameters $(\mathrm{C} 1, \mathrm{C} 2$, and $\mathrm{C} 3)$ as input values which could be Clinical signs, genetic information, age or biological results. Fuzzy rules were then applied on the concepts. If there is a good similarity between known cases and patient a conclusion is drawn, else an FCM is used for diagnosis and therapeutic decision.

In a related work by [13], Fuzzy logic was used to detect heart disease using 6 input fields (chest pain, blood pressure, cholesterol, blood sugar, maximum heart rate, old peak) and 2 output fields (result, and precautions) with rule base that consist 22 rules. The output detects the presence of heart disease and precautions accordingly. And the observed result was $92 \%$ accurate.

\section{3) Artificial neural network (ANN)}

In a study by [14] neural networks and decision tree were used to develop a patient specific real time alarm algorithms. They observed that alarms are built retrospectively by dataset that encompass thousands of patients to detect adverse clinical and medical conditions. But without further improvement, studies found that a model built from one patient population has worse performance on data from other two populations. So, their research explores building patient-specific alarming models from a specific individual models data. Of the two algorithms employed in the research (Decision Tree and Neural Network), Neural Network was recommended because it has a higher accuracy rate $(96 \%$, $99 \%, 79 \%$, and $99 \%$ ). However, the result from Decision Tree $(84 \%, 98 \%, 72 \%$, and $98 \%$ ) was not so bad, just that it was not good enough compared to the ones from neural networks.

Data from 11 different patients of various ages was collected from 196 hours. And neural network of single output layer with one hidden layer was used with two different training times i.e. 2 and 8 hours. Although the performance of the 2 hours trained network was poor, the 8 hours trained network was considered very well because it outperforms the generic alarm algorithms in devices.

\section{B. Achievement of Accurate Diagnosis}

The importance of accurate diagnosis need not be overemphasized in the medical field, because improper or wrong diagnosis results in serious implications for health outcome. This problem varies from over-diagnosis to under-diagnosis resulting in the next problem to be discussed later in this paper i.e. treatment optimization. However, the AI algorithms used to address this problem i.e. achieving accurate diagnosis, are discussed in this section.

\section{1) Artificial neural network (ANN)}

Several studies show the ability of ANN to accurately diagnose some diseases e.g. malignant melanoma, eye problems, and different forms of cancer by analyzing diagnostic criteria and spectral information. The application of ANN in medical diagnostics varies; one of the ways the ANN is applied as stated by [15] is shown in Fig. 3.

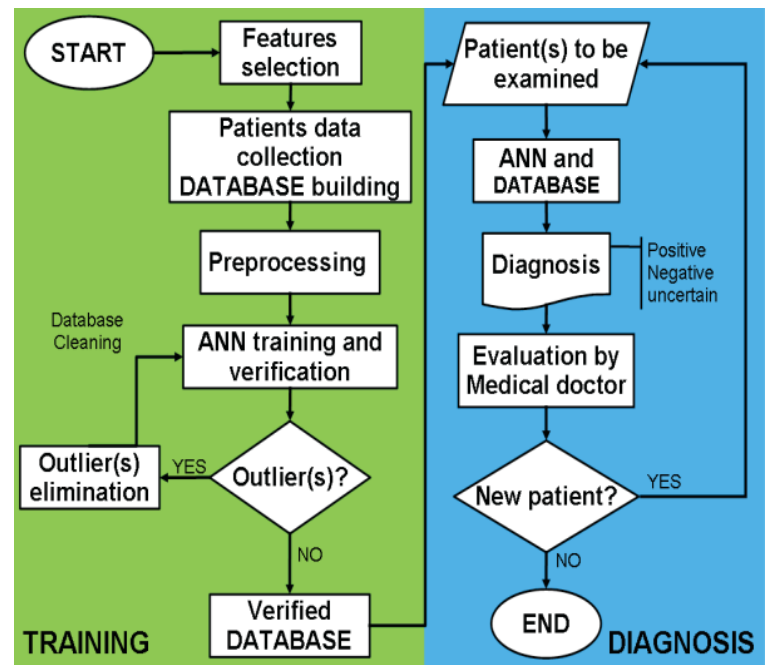

Fig. 3. Fundamental steps in ANN-based medical diagnostic[15].

In a research by [16], ANN was applied to diagnose kidney stones. The research was conducted on a dataset that consist 1000 patients records, and 7 attributes that were the actual symptoms of kidney stones. The research compared three neural network algorithms i.e. Radial Basis Function (RBF), Learning Vector Quantization (LVQ), and Multilayer Perceptron (MLP) with back propagation. Also, WEKA was used as a tool, with $70 \%$ training, $15 \%$ testing, and $15 \%$ validation.

The result shows MLP outperforms the other algorithms with an accuracy of $92 \%$ and correctly classified instances of 922, and incorrectly classified instances of 23.

\section{2) Expert systems}

A rule based Expert System was developed by [17] that produces relevant data and information for consultations, results, and possible diagnosis. Their research was scoped to Malaria, chicken pox, diarrhea, diabetics, cholera, asthma, jaundice, hepatitis, typhoid, thyroid, migraine, scatia, bronchitis, and Alzheimer's disease.

Also, [18] developed a rule based expert system that makes inference with symbols that require the translation of a specific knowledge in standard symbolic form. Data was collected that associate patients with sign/symptoms and diseases. The symptoms are organized in groups and the outcome is categorized into a suggestion, suspicion, or suspension of diagnostic ideas to the user (doctor).

\section{3) Support vector machine (SVM)}

In a related research, [19] implemented and intelligent system based support vector machine and Radial Basis Function (RBF) type of Artificial Neural Network (ANN) to diagnose heart disease. However, SVM was proposed because it produced a better result $(86.42 \%)$ compared to $\mathrm{RBF}(80.81 \%)$. A total medical record of 214 instances consisting 19 attributes and 4 class distribution were used. Binary classification was used in which 78 instances belong to the class ' 0 ' i.e. Myalgia meaning normal, while 139 are considered patients with heart problems. 


\section{Treatment Optimization}

\section{1) Artificial neural network (ANN)}

Several studies have been conducted to predict the clinical course of Graves Disease (GD) in patients treated with Antithyroid drugs. [20] Studied the clinical outcome of GD after Antithyroid therapy by defining a set of easily achievable variables able to predict, as early as possible. The study contained 71 patients with GD treated with Methimazole for 18 months: 27 of them achieved stable remission for at least 2 years after Methimazole therapy withdrawal, whereas 44 patients relapsed. Then, at first used Perceptron-like artificial neural network (ANN) to predict remission or relapse after Methimazole withdrawal. Twenty-seven variables obtained at diagnosis or during treatment were considered. Among different combinations, they identified an optimal set of seven variables available at the time of diagnosis, whose combination was useful to efficiently predict the outcome of the disease following therapy withdrawal in approximately $80 \%$ of cases. This set consists of the following variables: heart rate, presence of thyroid bruits, psychological symptoms requiring psychotropic drugs, serum TGAb and fT4 levels at presentation, thyroid-ultrasonography findings and cigarette smoking. Finally, they concluded that perceptron-like ANN is potentially a useful approach for GD-management in choosing the most appropriate therapy schedule at the time of diagnosis.

\section{2) Nä̈ve Bayesian}

In a related research by [21] on Medical treatment using data mining examining the relationship between diseases and their treatments. Also to identify diseases names and symptoms and extract the sentence containing that details from an article, then get the relationship that exists between the disease and the treatment. Naïve Bayesian algorithm was used on pre-processed text to classify the information into 'cure', 'prevent', and 'side effects' to patients. The data used for this research was a 'live' template.

\section{3) Support vector machines (SVM)}

In a related study, [22] developed a Hybrid Machine Learning (ML)-based methodology that is fused with an SVM classifier in combination with Bag-of-Words Representation and Natural Language Processing (NLP) tasks for building an application that is capable of identifying and disseminating healthcare information. This was implemented using two representative models of a public medical dataset containing 2700 record: Complement Naive Bayes (CNB), which is adapted for text with imbalanced class distribution with a linear classifier support vector machine (SVM) with polynomial kernel, and a classifier that always predicts the majority class in the training data (used as a baseline). The best results obtained are: 98 percent F-measure for the class Cure, 100 percent F-measure for the class Prevent, and 75 percent F-measure for the Side Effect class.

\section{FUTURE TRENDS}

Personalized Medicine is an active research area with a lot of envisioned products and achievements. Some of these future trends include:

Preventive Medicine: Preventive Medicine is a future trend related to personalized medicine. It is envisioned that, sooner or later, every inanimate object will be able to recognize your genetic code-including that taunting vending machine in your office, which may or may not advise you to skip the chocolate bar today, so that you can skip diabetes tomorrow [5].

Robotic Surgery: Researchers are currently working on developing robots that could perfectly perform surgery on humans. Robotic surgery such as da Vinci ${ }^{\circledR}$ help enables surgeons operate with enhanced vision, precision, dexterity, and control. Also, the use of robotic surgery remotely is envisioned in the new future; especially to carry out surgery on Astronauts. A fist-sized robot is scheduled for its first zero-gravity test in the next several months - one small step toward enabling robotic medical attention for humans stuck on deep-space missions lasting for months, [23]. Also, the use injected micro chips to perform surgical or organ correctional operations are envisioned instead of cutting out the human body.

\section{CONCLUSION}

The implementation of Personalized Medicine heavily relies on AI algorithms as discussed in this review. However, it is still in its early stage and levels and faces some challenges; some of which have a direct link to AI were discussed in this report. While other problems such as research and implementation costs, and government regulations are also challenges which are critical to the successful implementation of personalized medicine, but not addressed by the algorithms discussed in this report. However, Personalized Medicine does not only faces challenges; it does pose some challenges as well, such as; changing the medical profession and practice to the extent that some futurist think algorithms and machines could replace most of the jobs doctors do today. Finally, a successful implementation of personalized medicine would save many lives and perfect the medical profession.

\section{REFERENCES}

[1] W. Karen. (May 2013). GNS aims to help MDs know which treatment will work the best for each patient. The Boston Globe. [Online] Available:

http://www.bostonglobe.com/business/2013/05/12/personalized-medi cine-goal-big-data-scientist/28gTkXjCDj6Zh6KP5tpNBO/story.html

[2] B. U. Ozomaro, C. B. Nemeroff, C. Wahlestedt, A. Goal et al., "Personalized medicine and psychiatry: Dream or reality?" Psychiatric Times, pp. 1-6, 2014.

[3] G. S. Ginsburg and J. J. McCarthy, "Personalized medicine: Revolutionizing drug discovery and patient care," Trends Biotechnol., vol. 19, no. 12, pp. 491-496, Dec. 2001.

[4] N. Douali and M.-C. Jaulent, "Genomic and personalized medicine decision support system," in Proc. 2012 IEEE Int. Conf. Complex Syst., Nov. 2012, pp. 1-4.

[5] A. Katrin. (November, 2013). Let's get personal: Surfing the big waves in big data and personalized medicine. MaRS. [Online]. Available: http://www.marsdd.com/news-and-insights/lets-get-personal-surfing-b ig-waves-big-data-personalized-medicine/

[6] S. Galit, R. P. Nitin, and C. B. Peter, Data Mining for Business Intelligence: Concepts, Techniques, and Applications in Microsoft Excel ${ }^{\circledR}$ with XLMiner ${ }^{\circledR}, 2^{\text {nd }}$ Edition, John Wiley \& Sons, 2010, ch. 8 , pp. 102-104. 
[7] R. Bardan, Artificial Neural Networks. Applications in Urology, Department of Urology, Victor Babes University of Medicine and Pharmacy, pp. 289-296, 2004.

[8] I. Y. Khan, P. H. Zope, and S. R. Suralkar, "Importance of artificial neural network in medical diagnosis disease like acute nephritis disease and heart disease," International Journal of Engineering Science and Innovative Technology, vol. 2, no. 2, pp. 210-217, 2013.

[9] L. Auria and R. A. Moro, "Support vector machines (SVM) as a technique for solvency analysis," Discussion Papers of DIW Berlin, no. $811,2008$.

[10] S. Martin. Disadvantages of support vector machines (SVM). [Online] Available: http://www.svms.org/disadvantages.html

[11] Personalized medicine coalition: Issues affecting adoption of personalized medicine, American Society of Human Genetics, PMC PM issues 3, 2007.

[12] M. R. Kosorok, "Personalized medicine and artificial intelligence," $\mathrm{Ph} . \mathrm{D}$. dissertation, Department of Biostatistics University of North Carolina at Chapel Hill, pp. 1-50, 2012.

[13] K. J. Jang, G. Balakrishnan, Z. Syed, and N. Verma, "Scalable customization of atrial fibrillation detection in cardiac monitoring devices: Increasing detection accuracy through personalized monitoring in large patient populations," in Proc. IEEE Eng. Med. Biol. Soc. Conf., Jan. 2011, vol. 2011, pp. 2184-2187.

[14] S. Kumar and G. Kaur, Detection of Heart Diseases Using Fuzzy Logic, vol. 4, no. 6, pp. 2694-2699, 2013.

[15] Y. Zhang and P. Szolovits, "Patient-specific learning in real time for adaptive monitoring in critical care," J. Biomed. Inform., vol. 41, no. 3 , pp. 452-460, Jun. 2008.

[16] F. Amato, A. López, E. M. Peña-Méndez, P. Vaňhara, A. Hampl, and J. Havel, "Artificial neural networks in medical diagnosis," J. Appl. Biomed., vol. 11, no. 2, pp. 47-58, 2013.

[17] K. Kumar and A. Abhishek, "Artificial neural networks for diagnosis of kidney stones disease," Int. J. Inf. Technol. Comput. Sci., vol. 4, no. 7, pp. 20-25, Jul. 2012.

[18] D. Biswas, S. Bairagi, N. Panse, and N. Shinde, "Disease diagnosis system," International Journal of Computer Science \& Informatics, vol. 1 , issue 2, 2011.

[19] P. S. K. Patra, "An expert system for diagnosis of human diseases," International Journal of Computer Applications, vol. 1, no. 13, pp. $71-73,2010$.

[20] E. Orunesu, M. Bagnasco, C, Salmaso, V. Altrinetti, P. Del Monte, G. Pesce, M. Marugo, and G. S. Mela, "Use of an artificial neural network to predict graves ' disease outcome within 2 years of drug withdrawal," European Journal of Clinical Investigation, vol. 34, issue 3, pp. 210-217, 2004

[21] S. Ghumbre, C. Patil, and A. Ghatol, "Heart disease diagnosis using support vector machine," in Proc. International Conference on Computer Science and Information Technology, 2011, pp. 84-88.
[22] K. Srinivasarao, "Hybrid machine learning implementations for classifying disease-treatment relations in short texts," International Journal of Advanced Research in Computer Science and Software Engineering, vol. 4, no. 2, pp. 63-67, 2014.

[23] H. Jeremy. (April, 2014). Small robot surgeon designed to work inside astronaut's bodies. IEEE Spectrum. [Online]. Available: http://spectrum.ieee.org/tech-talk/robotics/medical-robots/tiny-robot-s urgeon-designed-to-work-inside-astronauts-bodies

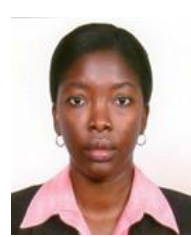

Rose Atuah received the bachelor degree in information communication technology from Ghana Institute for Management and Public Administration in 2011. She is currently pursuing a master degree in software technology at University Kebangsaan Malaysia with interest in software design and business process modeling.

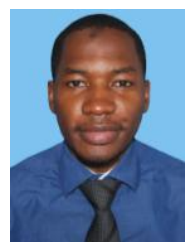

Ali Garba Garba received the bachelor degree in software engineering with multimedia from the Limkokwing University of Creative Technology in 2012 Currently he is pursuing the M.Sc. degree in information technology (industrial computing) at University Kebangsaan Malaysia. His main research interests include computer vision, machine learning and medical image analysis.

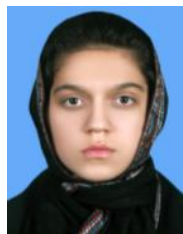

Anahita Ghazvini received the bachelor degree with honours in information technology (computer science) at Universiti Kebangsaan Malaysia (UKM) in 2013. Currently she is pursuing a master degree in information technology (artificial intelligence) at University Kebangsaan Malaysia (UKM) with interest in machine learning, image processing and computer vision.

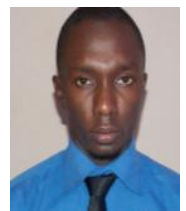

Jamilu Awwalu received his bachelor degree in business computing and information technology from the University of Wales in 2010. He is currently pursuing the M.Sc. degree in information technology (artificial intelligence) at University Kebangsaan Malaysia.

His research interests include opinion mining and machine learning. sentiment analysis, image processing, computer vision and 